\title{
Shunt scissors: technical note
}

\author{
Ralph G. Dacey, MD, ${ }^{1}$ Oliver E. Flouty, MD, ${ }^{2}$ M. Sean Grady, MD, ${ }^{3}$ Matthew A. Howard III, MD, ${ }^{2}$ and \\ Marc R. Mayberg, MD ${ }^{4}$
}

\begin{abstract}
${ }^{1}$ Department of Neurosurgery, Washington University, St. Louis, Missouri; ${ }^{2}$ Department of Neurosurgery, University of lowa Hospitals and Clinics, lowa City, lowa; ${ }^{3}$ Department of Neurosurgery, University of Pennsylvania, Philadelphia, Pennsylvania; and ${ }^{4}$ Department of Neurosurgery, University of Washington, Seattle, Washington
\end{abstract}

\begin{abstract}
OBJECTIVE When performing ventriculoperitoneal shunt surgery it is necessary to create a subgaleal pocket that is of sufficient size to accommodate a shunt valve. In most cases the valve is placed over the posterior skull where the galea begins to transition to suboccipital neck fascia. Dense fibrous attachments in this region of the skull make it technically awkward to develop the subgaleal valve pocket using standard scissors and a blunt dissection technique. In this report the authors describe a new device that enables surgeons to create the shunt valve pocket by using a simple semi-sharp dissection technique.
\end{abstract}

METHODS The authors analyzed the deficiencies of the standard valve pocket dissection technique and designed shunt scissors that address the identified shortcomings. These new scissors allow the surgeon to sharply dissect the subgaleal space by using an efficient hand-closing maneuver.

RESULTS Standard surgical scissors were modified to create shunt scissors that were tested on the benchtop and used in the operating room. In all cases the shunt scissors proved easy to use and allowed the efficient and reliable creation of a subgaleal valve pocket in a technically pleasing manner.

CONCLUSIONS Shunt scissors represent an incremental technical advance in the field of neurosurgical shunt operations.

https://thejns.org/doi/abs/10.3171/2017.6.JNS171108

KEY WORDS shunt scissors; ventriculoperitoneal shunt; valve; surgical technique

$\mathrm{T}$ HROUGHOUT the history of our specialty, neurosurgeons have engaged in a relentless quest to develop new surgical devices to improve patient outcomes. Some new devices, such as endovascular coils and deep brain stimulators, have proven enormously impactful and are now used to treat patients throughout the world. ${ }^{1,4}$ Other inventions represent important but incremental improvements, such as technical modifications that allow surgeons to manipulate microscope controls with their teeth or toes. ${ }^{2,3}$ Several factors influence the rate at which new devices and techniques are adopted by neurosurgeons. Cost and ease of use are key considerations. Another more difficult to quantify variable that impacts new device adoption is the technical esthetic sensibility of the individual surgeon.

When performing microsurgical procedures, for example, surgeon preferences vary for the types of instruments displayed on the operative field. Some surgeons prefer a broad range of instruments specifically designed to optimally address any technical challenge that might be encountered during the procedure. At the other end of the spectrum, some neurosurgeons take a more workmanlike approach to performing procedures. They are content to use a small complement of instruments to overcome any and all technical challenges encountered, regardless of how effortful the process might be.

In this brief technical note, we describe a new device and method designed to refine one small but important step in the ventriculoperitoneal shunt (VPS) operation. Specifically, we describe shunt scissors that are designed to enable surgeons to gracefully create a subgaleal shunt valve pocket in an efficient and technically pleasing manner.

\section{Methods}

When a VPS procedure is performed, it is necessary to create a subgaleal pocket that will accommodate the shunt valve. Under most circumstances the valve will be positioned over the posterior skull. In the case of a posterior VPS, the pocket is positioned just caudal to the posterior bur hole, in line with the planned path of the subcutane- 
ously placed peritoneal catheter. There is increased mechanical resistance to dissection and development of a subgaleal plane in this region of the scalp, due to the transition that occurs between the galea of the posterior skull and suboccipital neck fascia. This increased resistance is particularly pronounced in large adult male patients.

\section{Surgical Technique}

The current technique for developing the subgaleal valve pocket involves inserting standard surgical scissors into the subgaleal space while the instrument is in the closed position, and then spreading the blades open to expand the space and create the pocket. This is a surgical scenario in which the instrument being used is not designed optimally for the technical task that must be performed. Surgeons are simply making do with an existing device designed for a different purpose to create the pocket, even if it requires inelegant application of large manual forces.

\section{Device Design}

Our group performed a systematic analysis of the current technique and identified 2 fundamental device design and workflow flaws. First, standard scissors have their sharp cutting surfaces on the wrong side of the blade for this clinical application. The tissue that needs to be cut to create the shunt valve pocket abuts the outside surfaces of standard scissors blades, and that tissue is too adherent to the skull to separate easily when applying a blunt dissection technique. Second, the dissection motion with standard scissors requires that the surgeon spread apart the scissors handles, rather than using a more easily controlled and powerful closing motion. Throughout their training and subsequent practice, neurosurgeons learn to perform sharp dissecting maneuvers with scissors by approximating the fingers (e.g., cutting sutures or using microscissors to cut fine structures), not spreading them apart. A scissors-opening motion is well learned and effective when applied to a blunt dissection technique when there is little mechanical resistance to dissection, but we reasoned that a closing motion would be more suitable for an instrument used to perform sharp dissection.

The shunt scissors were created to address both design deficiencies in a simple instrument that is easy and intuitive to use. We modified standard surgical scissors so that the cutting blade surfaces face outward, and the bladehandle axes are offset such that the blades are separated when the scissors are in the "blades open" position (Fig. 1). Cardboard cutouts scaled to standard surgical scissors blades were used to test how different blade angle offsets at the hinge point would impact the size of the resulting shunt pocket. We found that an offset angle of $40^{\circ}$ resulted in the optimal size for a shunt pocket for standard shunt valves in adults, and we produced shunt scissors using this design. The subgaleal pocket is created by inserting the closed blades of the shunt scissors into the subgaleal space, and then spreading the blades apart by closing the handles together (Fig. 2).

Shunt scissors prototypes were manufactured in the University of Iowa medical instruments machine shop. They were then tested on the benchtop by 4 experienced

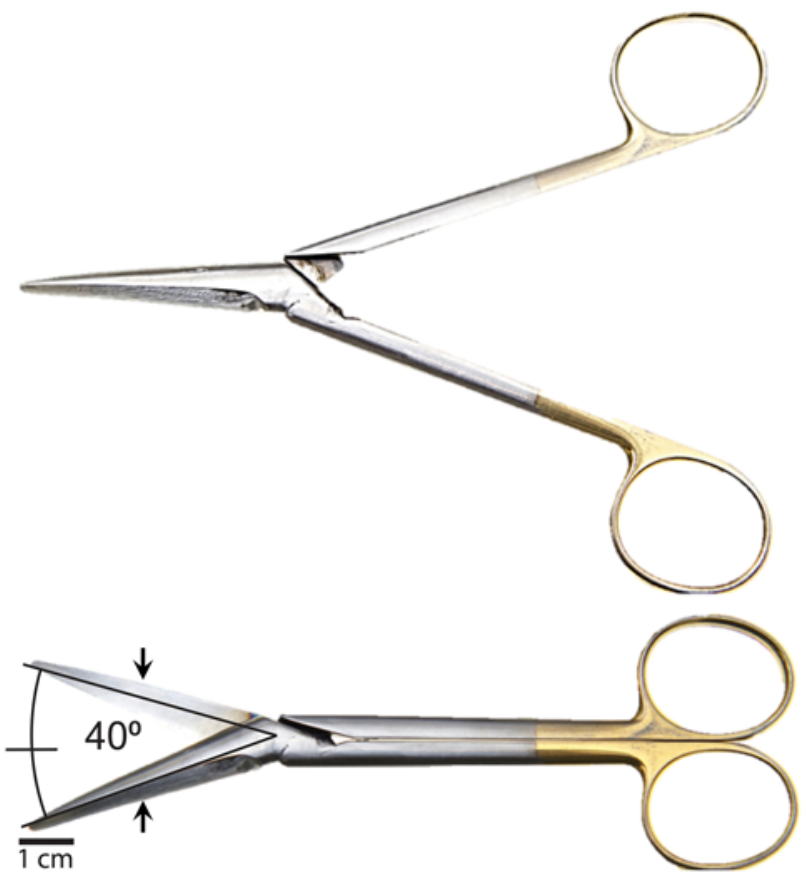

FIG. 1. Photographs of shunt scissors in the blades-closed and bladesopen configurations. The handle and blade are angularly offset so that the blades are separated by $40^{\circ}$ in the blades-open position when the handles are closed.

faculty neurosurgeons practicing in the Midwest, West Coast, and East Coast of the US, and were later used intraoperatively. Representative intraoperative images in Fig. 2 show the device in use during a posterior VPS operation.

\section{Results}

Each neurosurgeon provided feedback about his or her experiences and impressions with the shunt scissors. Nationally there were no regional differences in the capacity of surgeons to master the use of this new device. All surgeons initially found the unusual mechanical relationship between the movement of the scissors handles and blades counterintuitive. However, with a few minutes of benchtop practice this barrier was overcome. After that period of adaptation, in all clinical cases the shunt scissors were easy to use and highly effective at creating an adequately sized valve pocket in less than 5 seconds per case. During the initial study period the shunt scissors were used in 10 adult patients during de novo shunt surgery placement. Eight operations were posterior and 2 were frontal catheter placements. In the later cases the shunt scissors were used to create a shunt valve pocket adjacent to a linear postauricular incision. There were no complications related to the use of shunt scissors, and subjectively it seemed that less manual force was needed to create the pocket compared with traditional blunt dissection methods.

\section{Discussion}

In this report, we describe a simple device and technique for creating a subgaleal valve pocket during VPS surgery. As with any neurosurgical procedure, attention must be 

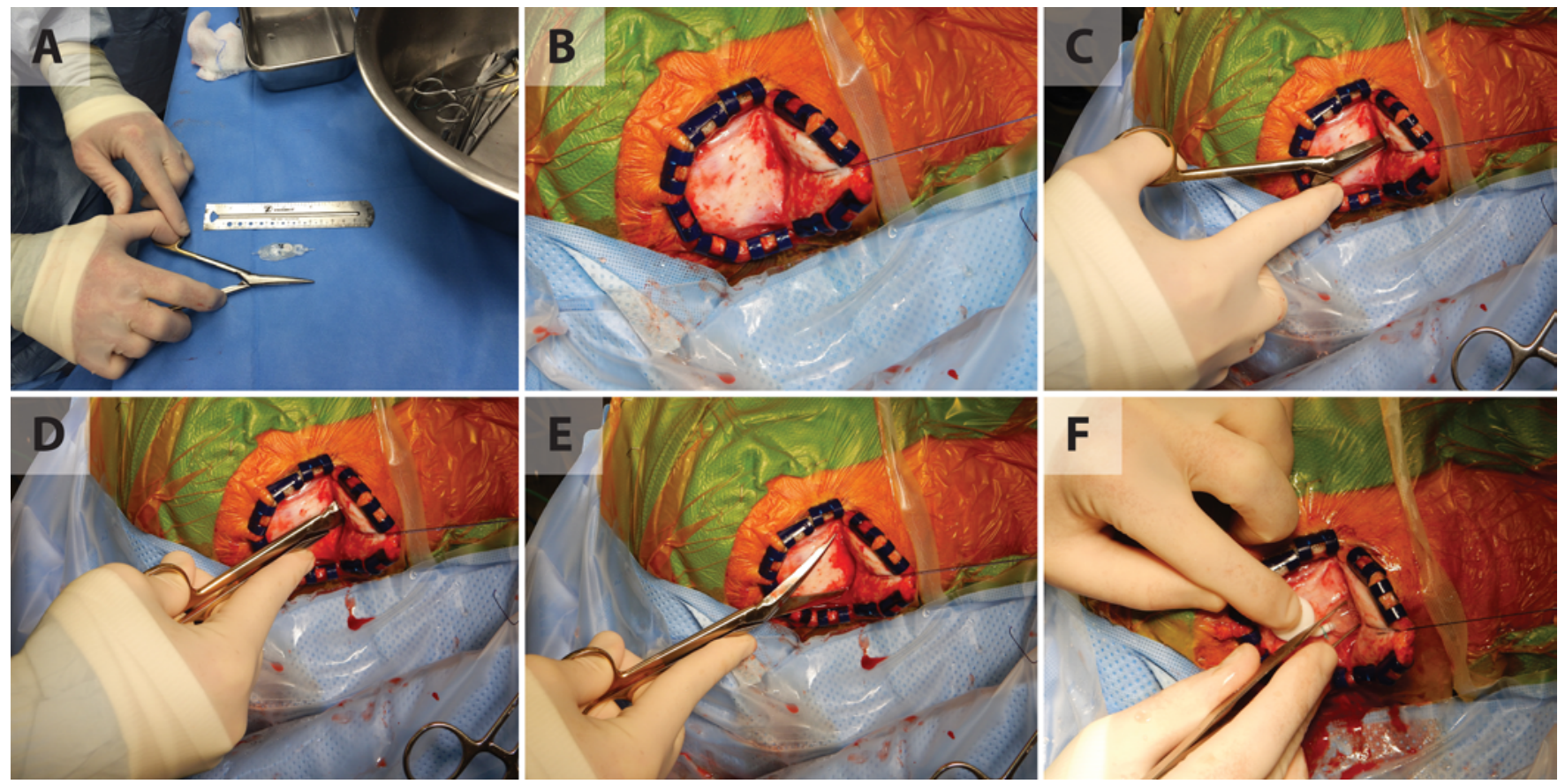

FIG. 2. Intraoperative photographs of the shunt scissors being used in a representative posterior VPS placement procedure. A: The surgeon confirms that the blades of the scissors are appropriately scaled for the shunt valve that will be placed. B: A posterior scalp flap is created, exposing the subgaleal space. C: Scissors are advanced into the subgaleal space while in the blades-closed position. D: The scissor blades are opened to create the subgaleal pocket by closing the handles. E: Scissors are withdrawn while in the blades-open position, insuring unobstructed access to the subgaleal pocket. F: The shunt valve is advanced into the subgaleal pocket.

paid to all technical details of a shunt operation to achieve optimal outcomes. In the case of shunt surgery, if the valve pocket is too small or inappropriately positioned, the shunt system can be implanted in a manner that results in kinking and obstruction. When standard scissors are used to create the pocket, particularly in large adults, it is sometimes necessary to use forceful and technically awkward movements to break through the connective tissue within the posterior subgaleal space. A periosteal elevator can also be used as a blunt dissection instrument for this purpose. Shunt scissors address this technical problem by allowing the surgeon to use a semi-sharp, rather than blunt, dissection technique with the aid of a simple, easy-to-use device.

\section{Conclusions}

Shunt scissors effectively address a minor technical limitation of the current VPS operation. The device is inexpensive and easy to use, and could in theory reduce the risk of a rarely encountered complication.

\section{Acknowledgments}

We thank Mike Neville from the University of Iowa medical instruments machine shop. We also thank the former neurosurgery residents who helped draw attention to the importance of creating an adequate subgaleal valve pocket during posterior VPS procedures.

\section{References}

1. Bronstein JM, Tagliati M, Alterman RL, Lozano AM, Volkmann J, Stefani A, et al: Deep brain stimulation for Parkin- son disease: an expert consensus and review of key issues. Arch Neurol 68:165-171, 2011

2. Holly EH: Mouth guide for operating microscope. Technical note. J Neurosurg 44:642-643, 1976

3. Kobayashi S, Sugita K, Matsuo K: An improved neurosurgical system: new operating table, chair, microscope and other instrumentation. Neurosurg Rev 7:75-80, 1984

4. Molyneux AJ, Kerr RSC, Yu LM, Clarke M, Sneade M, Yarnold JA, et al: International subarachnoid aneurysm trial (ISAT) of neurosurgical clipping versus endovascular coiling in 2143 patients with ruptured intracranial aneurysms: a randomised comparison of effects on survival, dependency, seizures, rebleeding, subgroups, and aneurysm occlusion. Lancet 366:809-817, 2005

\section{Disclosures}

Dr. Grady is a consultant for Integra and an investigator for the National Institute of Neurological Disorders and Stroke (NINDS).

\section{Author Contributions}

Conception and design: Howard, Dacey, Mayberg. Acquisition of data: all authors. Analysis and interpretation of data: all authors. Drafting the article: all authors. Critically revising the article: all authors. Reviewed submitted version of manuscript: all authors. Approved the final version of the manuscript on behalf of all authors: Howard. Administrative/technical/material support: Howard, Dacey, Mayberg. Study supervision: Howard, Dacey, Mayberg.

\section{Correspondence}

Matthew A. Howard III, University of Iowa Hospitals and Clinics, 200 Hawkins Dr., Iowa City, IA 52242. email: matthew-howard@ uiowa.edu. 